\title{
The Research of Irregular Volume Calculation Methods for Vivo Organs
}

\author{
Zhizun Zhou* Hongyu Zhang Yanfang Yang \\ Imaging Faculty \\ Mudanjiang medical university \\ Mudanjiang city, Heilongjiang province, P. R. China \\ E-mail: zzho007@hotmail.com
}

\begin{abstract}
Objective The human living organ irregular volume calculation and 3D imaging method will give a potential hope for operation direction, tumor spatial location fixing. This project will also find a new method to study human brain volume calculation and this can enhance the neuroscience development. Methods On the base of threshold segmentation, the methods of organ gradient setting and volume data transparent visualization were given. Those can be use to draw image fast and accurately. They can also use to detect inner organ information of human body and measured organ structure. Result and Conclusions By using those methods, the 3D reconstruction image of brain, hart, kidney and liver were formed. From those images the internal organs of body can be observed clearly. The volume ratio of grey matter and white matter, the volume of hart, kidney, liver and tumor position were derived and measured.
\end{abstract}

Keywords-component; threshold segmentation; $3 D$ reconstruction; vivo organ volume

\section{INTRODUCTION}

In medical image 3D visualization technology, medical volume data shows the organ internal situation. There is great significance to positioning the lesion, diagnosis and treatment. Reconstruction of the target structure of the boundary surface can help to show the tissue of threedimensional images contains. More importantly, this method can realize living tissue structure, quantitative measurement [11, 12]. The introduced construction and representation method of medical volume data in MATLAB in the first part were introduced in this article. The geometric information in the volume data was describes. The method provided a theoretical basis for quantitative measurement of organ structure. The second section discusses tissue volume data analysis based on twodimensional image threshold segmentation method [13, 14]. The third section focuses on the morphological image processing techniques used MATLAB implementation standards to threshold segment volume data set for postprocessing and remove most non-interested organizations. The fourth section describes the gradient setting method of the tissue volume data partition reorganization, multi-layer equivalent surface extraction method and its gradient transparent visualization technology. Applying above research methods for three-dimensional visualization images and its qualitative, quantitative image measurement and analysis results were finally achieved, and the advantages and advantages of those methods were discussed as well.

\section{PRINCIPLE AND METHODS}

In the medical field, a variety of medical imaging equipment such as CT, MRI or ultrasound human organ different layer scans can obtain a set of consecutive tomographic images after the image reconstruction. A tomographic image is an arbitrary two-dimensional digital images, $f(x, y)$, with representation in MATLAB(a kind of software use for medical image processing), can be naturally expressed as a two-dimensional matrix:

$f[x, y]=\left[\begin{array}{cccc}f(1,1) & f(1,2) & \Lambda & f(1, N) \\ f(2,1) & f(2,2) & \Lambda & f(2, N) \\ \mathrm{M} & \mathrm{M} & \mathrm{M} & \mathrm{M} \\ f(M, 1) & f(M, 2) & \Lambda & f(M, N)\end{array}\right]$

The matrix $M \times N$ represents the size of this medical two dimensional image, and $x, y$ are space (plane) coordinates, $(1,1)$ is origin point, and $f$ represents the value of certain properties of the image at the point where. For the grayscale image, the grayscale values $f$ represent human tissue physical properties. Since the coordinate values $(x, y)$ are discrete, so that the nature of the space is discrete, that is, by sampling, making human tissue physical properties quantized [3].

A group of consecutive CT, MRI scan tomographic images of the same nature will be ordered to read into a three-dimensional matrix to achieve the construction of medical volume data, with representation by $D(x, y, z)$ in MATLAB. The basic data type abstract is fundamental research of three-dimensional visualization of medical imaging techniques [8].

The nature of each tomographic image is a medical volume data may be determined by discrete samples of physical properties limited space, and can be expressed as: 


$$
f(x), x \in R^{n},\{x\}
$$

It is a collection of $n$ dimensional space of sampling points, and therefore the volume data can be seen as a data set. When the medical image data set can be abstracted into a three dimensional structural scalar volume data [9], the three-dimensional array is expressed as:

$$
\{D[x, y, z],(\Delta x, \Delta y, \Delta z)\},\left\{\begin{array}{l}
x=1,2, \mathrm{~L}, M \\
y=1,2, \mathrm{~L}, N \\
z=1,2, \mathrm{~L}, P
\end{array}\right\}
$$

Each pixel is abstracted as a sampling point, and $\Delta x, \Delta y, \Delta z$ are the distance of sample points on the three axes, and therefore, $\Delta x \times \Delta y$ in space (in general) to the pixel area (Pixel Spacing) are equal, $\Delta z$ representing the layer spacing (Slice Thickness) $M, N, P$ Is the dimension of the volume data, and $M \times N$ indicates the size of each piece of the tomographic image. It is generally present as $512 \times 512, P$ is usually represent hundred scan layers. $D[x, y, z]$ is referred to as three-dimensional volume data or density of the gradation data on the sampling points. 8 adjacent sampling points constitute a rectangular cube. The volume data is called a primitive voxel. $\Delta x \times \Delta y \times \Delta z$ representative of the volume of the voxel size and all cubes constitute a continuous the sample area of three-dimensional image, and therefore, the volume of solid areas of the threedimensional region can be used to estimate voxel count [3].

Before to analyze the image data set, the strict alignment of these image sequences and image registration must be solved first, others, the reconstruction results will appear misplaced. For simplistic interpretation of the tissue volume data segmentation method, the multi-threshold tissue segmentation method based on a single twodimensional histogram analysis was described first. Select an image features in the volume data which scanned images are normal MR head cross-sectional layers, the MR structure can be divided into air, cerebrospinal fluid, brain gray matter, white matter, scalp and skull parts.

For probe gray value and visual inspection of the histogram of tissues to achieve ideal results intuitively and simply, tissues of image intensity range value (i.e. the lower and upper limits of the gray level threshold) in the interactive environment by manually selecting high-quality images were divided. The value of this range should include most of the pixel gray value of tissues $[1,2]$. Due to the different imaging principle, for CT image, the size of the pixel gray values directly reflect the tissue density. MR image does not have a density similar. Usually soft tissue has a higher gray value and gray histogram can still be distinguished from different tissues of the MRI.
Two-dimensional image was constructed by the different tissue types (cluster number $K$ ) component and each pixel extracted from the cluster, subordinated to a normal distribution for each cluster the pixel gray, with the mean ${ }^{\mu}$, standard deviation $\sigma$ and the number of pixels $n$ description. Image $f(x, y)$ of multi-mode (i.e. peak) frequency curves were derived from the tissue type proportion of distribution by a probability density function weighted fitting $[3,4]$, using the following form:

$$
h(f)=\sum_{i=1}^{K} \frac{P_{i}}{\sqrt{2 \pi \sigma_{i}}} \exp \left|-\frac{\left(f-\mu_{i}\right)^{2}}{\sigma_{i}^{2}}\right|
$$

$h(f)=P_{i}$, represent priori probability of a certain specific pattern, so $P_{i}=1$. Histogram of the peak number reflects contains approximate number of tissues [8]. Valley, the two probability density function intersection was dividing line between the different tissues. Two-dimensional image histogram analysis can identify one or more threshold to split tissues.

The two thresholds, $\left(T_{1}, T_{2}\right)$ are identified for threedimensional grayscales image threshold segmentation, set input image is $D(x, y, z)$, and output images is $D^{\prime}(x, y, z)$, then

$D^{\prime}(x, y, z)=\left\{\begin{array}{cc}D(x, y, z) & T_{1} \leq D(x, y, z) \leq T_{2} \\ 0 & \text { others }\end{array}\right.$

Using MATLAB the volume data set of all topographic image sequences can be easily be segmented when the lower and upper thresholds was determined. All other threshold range of the forthcoming volume data elements grayscale value is set to $0[5,6]$. The advantages of this segmentation technique are to split the complex spatial simplified into a simple threshold process. Fig .1 is original image, which is choose from opening medical database. Fig .2 is a threshold segmentation of brain parenchyma grayscale image which was determined by using this model.

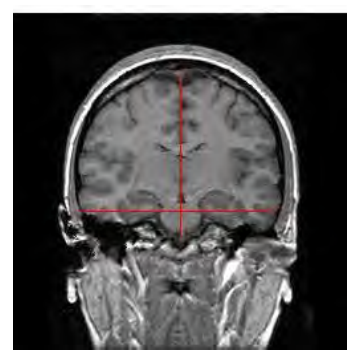

Figure 1. the original image 


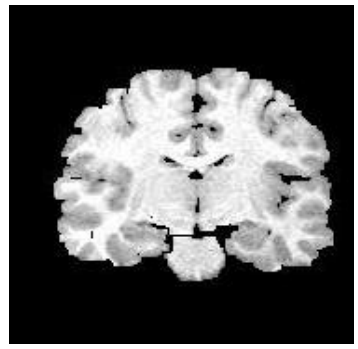

Figure 2. threshold segmented brain gray level image

\section{EXPERIMENTAL RESULTS AND ANALYSIS}

An important organ or tissue structure in the twodimensional image was show in Fig .3. MATLAB, Photoshop software were applied to realize interactive segmentation, using surface rendering method for threedimensional reconstruction. The reconstruction structure can be displayed alone, overall rotating three-dimensional space and different structures can also be transparent display that clearly shows the spatial position relationship of organs. Three-dimensional model of the organ can be established as well. Using Photoshop software, the brain and scalp junction of the main structure were finely semi-automatic segmented [9]. Surface rendering reconstruction can be fast realized by Data which were divided in three-dimensional visualization software on the PC or graphics workstation [10]. Due to the high spatial resolution, 64-slice spiral CT has a unique advantage in the field of three-dimensional reconstruction of craniofacial bone, viscera and other organs. 3.0T ultra high-field MRI has unique advantages in the field of three-dimensional reconstruction of brain tissue and can quickly acquire entire organ medical information. In this study, the head and organs of normal volunteers and patients were scanned, to ensure the reconstruction of threedimensional geometric model has a good simulation, display the fine structure and accessed to 400-500 tomography. The three-dimensional reconstruction was done respectively to obtain three-dimensional geometric model of the normal volunteers and patient skull, brain tissue and other vital organs.

Using MATLAB measurement tools we measured the liver, spleen of average length, width, thickness to determine the cirrhosis patients lesions such as tumors, the density anomaly and analyze the diseased tissue threedimensional positioning. To achieve the organizational structure of high-precision quantitative measurements, the size of the organs and tissue can be calculated based on the number of voxels and voxel size of the different anatomic structure of the human in the process of this algorithm.

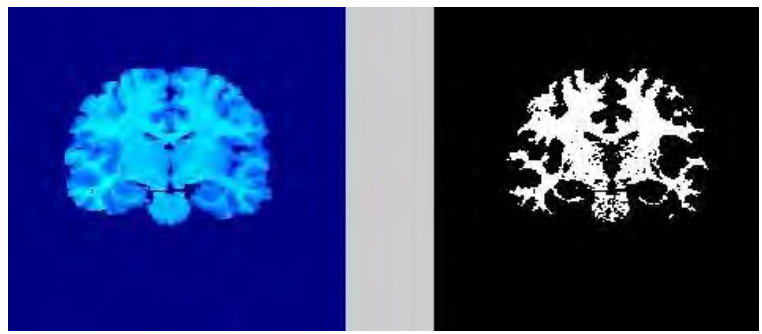

Figure 3. brain volume measurement

This algorithm of boundary target structure reconstruction can clearly show the content of the threedimensional image and, more importantly, can realize tissue structure by using these high quantitative measurement techniques. The tissue object in the brain parenchyma volume is estimated by the number of voxels multiplied by the voxels volume. Voxel numbers of human anatomical structure can be calculated by different grayscale value of the tissue volume data in the algorithm. Table 1 shows the measurement data of the tissue object brain parenchyma volume in Fig .2.The white matter and cerebral gray matter volume ratio is $7: 10$. When explain the nature of the tissue of certain physical and (or) function using digital image measurement results, digital image in time, space and value of discrete reason have small differences between the measurement results and real human body [1,7]. Using our measurement method, the data obtained in Table I. The brain volume is $1.0425 \mathrm{dm}^{3}$. Cerebral gray matter volume is $0.6120 \mathrm{dm}^{3}$. White matter volume is $0.4306 \mathrm{dm}^{3}$. White matter and cerebral gray matter ratio is 0.7036 . Brain gray matter and entire brain volume ratio is 0.5870 . White matter and the entire brain volume ratio are 0.4130 .

The cerebral gray matter surface sulcus has irregular morphology. There are individual differences. In the twodimensional cross-section image, it is difficult to determine the location of a particular sulci and gyri of lesion or tissue of particular sulcaland gyri. White matter three-dimensional imaging technology value in our country has not been understanding and attention. This non-invasive, simple, practical study can clearly shown in vivo cerebral gray matter with its internal three-dimensional structure of the white matter of the brain surface. Ti will help for clinical lesions positioning, surgical guidance and judgment of brain tissue abnormalities. The article also provides brain tissue volume measurement methods to promote neurological diseases study. 
TABLE I. BRAIN VOLUME DATA

\begin{tabular}{|c|c|c|c|c|}
\hline \multirow{2}{*}{$\begin{array}{c}\text { Brain } \\
\text { Volume } \\
\text { data }\end{array}$} & \multicolumn{4}{|c|}{ experimental medical DICOM volume data } \\
\hline & $\begin{array}{c}\text { voxel } \\
\text { volume }\left(\mathrm{dm}^{3}\right)\end{array}$ & $\begin{array}{c}\text { voxel } \\
\text { number }\end{array}$ & $\begin{array}{c}\text { object } \\
\text { volume } \\
\left(\mathrm{dm}^{3}\right)\end{array}$ & $\begin{array}{l}\text { parenchyma } \\
\text { volume ratio }\end{array}$ \\
\hline $\begin{array}{c}\text { parenchyma } \\
S_{1}+S_{2}\end{array}$ & 2.2156 & 470548 & 1.0425 & ----- \\
\hline $\begin{array}{c}\text { gray matter } \\
S_{1}\end{array}$ & 2.2156 & 276204 & 0.6120 & 0.5870 \\
\hline $\begin{array}{c}\text { White } \\
\text { matter } S_{2}\end{array}$ & 2.2156 & 194344 & 0.4306 & 0.4130 \\
\hline
\end{tabular}

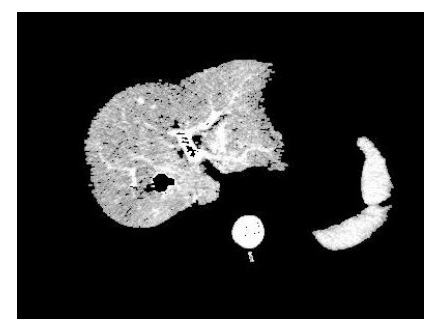

Figure 4. liver, tumoue, born and portal vein segmentation

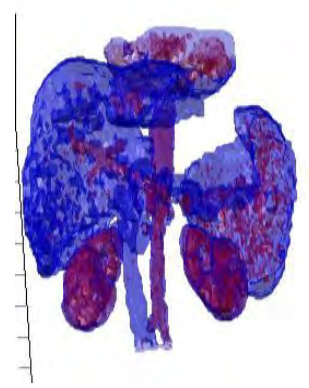

Figure 5. transparent organ $3 \mathrm{D}$ image

Selecting original volume data, a 46-year-old female liver cancer patient with liver CT enhancement, scanning portal vein of transverse sections, and the image has a significance characteristic. The abdomen of the experimental medical DICOM volume data from the Hongqi Hospital, Mudanjiang Medical university 64-slice spiral CT enhancement scan. Volume data are constituted by abdominal 64 cross-sectional layers. The interlayer spacing is $5 \mathrm{~mm}$. The pixel pitch is $0.732 \mathrm{~mm}$. Two-dimensional image size is $512 \times 512$. Volume data size is $24,117,248$ bytes, stored in 16 three-dimensional matrix D. Tumor size can be calculated to12 $\mathrm{mm} \times 13 \mathrm{~mm}$ by pixel pitch. Fig .3 shows the situation after segmentation of each image. Fig .4 is a cirrhosis patient abdominal enhanced CT scan of the artery phase volume data. The various organs of the abdominal three-dimensional reconstruction images were obtained by the algorithm. Using MATLAB measurement tools we can easily measure the liver, spleen average length, from the enlargement of width, thickness cirrhosis and hepatosplenomegaly were determined. Certain lesions such as tumors, abnormal density and rich blood supply can be three-dimensional positioned by using 3D visualization techniques from abnormal color region. In Fig .4, from the left hepatic lobe appeared unusually high density red clumps, we can fully determine the rich blood supply area is diseased tissue in the arterial phase.

Use MATLAB measurement tools, we can easily measure the intrahepatic tumor size is $12 \mathrm{~mm} \times 13 \mathrm{~mm}$ and small cancer foci vivo data is $167.9,212,38$.

\section{DISCUSSION}

The significance of the Algorithm and the threedimensional model in this subject is that doctors can use their own personal computer to implement patient's image data, standard three-dimensional visualization, image measurement and analysis, allowing physicians more convenient, intuitive and accurate study and improve clinical results. So that doctors can quickly generate realistic three-dimensional images on a PC, the interested region and its surroundings have been observed at the same time and precisely calculated vivo organ, diseased tissue volume, such as the cerebral gray matter and white matter volume, as well as their volume ratio and the vivo liver volume. Analysis of human vivo organs, the morphological changes of the diseased tissue will help doctors in the diagnosis, surgery, radiation oncology treatment. The threedimensional visualization algorithms can quantitatively and three-dimensional positioning analyzes disease tissue.

\section{ACKNOWLEDGMENT}

This research was supported by 2014Science and Technology Foundation of Heilongjiang Province education department No.12541846 and Heilongjiang Province university student creation practice Foundation No.201310229012.

* Corresponding author: Zhou Zhi-zun.

E-mail: zzho007@hotmail.com.

\section{REFERENCES}

[1] Zhou Zhi Zun, Xu Chun-Huan, Qiu Hui, "Measurement of the skull and its Comparative Study," J. Chinese Journal of Medical Physics, 2007, 9, 24 (5): 346-348.

[2] Ma Yu, Wang Lisheng, Tang Yuan Yuan, "Stepped edge surfaces in three-dimensional image tracking algorithm," J. Computer Aided Design and Computer Graphics, 2007, 19 (3) : 329-333.

[3] Doyle BJ, Morris LG, Callanan A, Kelly P, Vorp DA, McGloughlin $\mathrm{TM}$, "3D reconstruction and manufacture of real abdominal aortic aneurysms: from CT scan to silicone model," J. Biomech Eng. 2008;130(3): 34-50.

[4] Wu Shen, "Known extraction and semi-transparent visualization of three-dimensional image of the boundary surface," J. Computer Engineering, 2009, 35 (21) : 203-205.

[5] Yeh Ming, Yang Qingming, Li Niu, Wang Tao, "Fault medical image processing technology in orthopedic surgery," J. Journal of Shanghai Jiaotong University, 2003, 37 (1) : 30-33.

[6] Goshtasby A, Sonka M, Udupa J K, “Analysis of Volumetric Image," J. Computer Vision \& Image Understanding, 2000,77(2): 79-83. 
[7] Thomas Gerstner, "Fast multi-resolution Extraction of Multiple Transparent Iso-surfaces," J. Proceedings of VisSim 2001, Annual Conference, (3): 35-44

[8] Rodríguez JF, Ruiz C, Doblaré M, Holzapfel GA, "Mechanical stresses in abdominal aortic aneurysms: influence of diameter, asymmetry, and material anisotropy," J Biomech Eng. 2008;13(2): 21-23.

[9] J. Diepstraten, D. WeiskopF, T. Ertl, “Transparency in Interactive Technical Illustrations," J, Computer Graphics Forum, 2002, 21(3) : 317-325.

[10] Richard S.T, Frackowiak, “ Image Segmentation Human Brain Fuction,”. Beijing: Science Press, 2006: 698-700.
[11] Rafael C, Gonzalez, Richard E, "Woods, Steven L, Digital Image Processing Using MATLAB,". Beijing: Electronic Industry Press, 2008: 261-273.

[12] Lorensen WE, Cline HE, "A high resolution 3D surface construction algorithm," . Proceedings of the $14^{\text {th }}$ Annua, Conference on Computer Graphics and Interactive Techniques[C]. New York: ACM,1987. 163-169.

[13] Kim M, Taulbee DB, Tremmel M, Meng H, "Comparison of two stents in modifying cerebralaneurysm Hemodynamics," J. Ann Biomed Eng. 2008; 36(5): 726-41.

[14] Doyle BJ, Callanan A, McGloughlin TM, "A comparison of modelling techniques for computing wall stress in abdominal aortic aneurysms," J. Biomed Eng ,2007; 19(6): 38-42. 